\title{
Propuesta metodológica para analizar la carta del restaurante en base a la matriz Arthur D. Little
}

\author{
Methodological proposal to analyze restaurant menus \\ based on the Arthur D. Little Matrix
}

(iD) Rodrigo E. Riquelme Barros $₫$

Universidad Le Cordon Bleu. Lima, Perú

\begin{tabular}{llll}
\hline Recibido: 23/12/2019 & Revisado: 17/02/2020 & Aceptado: 02/04/2020 & Publicado: 18/06/2020 \\
\hline
\end{tabular}

\section{RESUMEN}

La ingeniería del menú es un conjunto de herramientas claves para evaluar la carta del restaurante. Durante mucho tiempo se ha pensado que la Matriz de Boston Consulting Group (BCG) es la única herramienta que permite hacer dicha evaluación, pero existen otros instrumentos que logran analizar de una manera más amplia la permanencia o eliminación de platos de la carta o menú. Hasta el momento, además de la Matriz BCG, podemos usar las siguientes herramientas: Ingeniería del precio (principios de Omnes), Índice de rentabilidad por plato (IRP), Índice de popularidad, Matriz de Pávesic, Matriz de Miller y el análisis Multicriterio. En este artículo se propone incorporar la Matriz de Arthur D. Little (ADL) ya que viene a complementar el análisis de la carta de los restaurantes al observar dos conceptos complementarios: las cantidades vendidas y el ciclo de vida de los productos (margen de contribución). Para lograr que la matriz ADL sea un aporte a la Ingeniería del menú, se propone hacer una reestructuración de esta matriz con la explicación y resultados obtenidos con el objetivo de tener mejores herramientas al momento de decidir la distribución, selección, permanencia y eliminación de los platos de la carta.

Palabras clave: Ingeniería del menú, matriz ADL, carta del restaurante.

\begin{abstract}
Menu engineering is a set of key tools to evaluate the restaurant menu. For a long time it was thought that the Boston Consulting Group (BCG) Matrix was the only tool to enable to do this evaluation; but there are other instruments that help analyze the permanence or removal of dishes in the menu. So far, in addition to the BCG Matrix, we can use the following tools: Cost engineering (Omnes principles), profitability index per dish (PID), popularity index, Pavesic Matrix, Miller Matrix, and the multi-criteria analysis. In this article, we propose to use the Arthur D. Little (ADL) Matrix as it complements the analysis of the restaurant menus by observing two complementary concepts: the amounts sold and the product shelf life (contribution margin). In order for the ADL matrix to contribute to menu engineering, we propose to re-structure this matrix with the explanation and results obtained so as to have better tools at the time of deciding the distribution, selection, permanence or removal of dishes in the menu.
\end{abstract}

Keywords: Menu engineering, ADL matrix, restaurant menu. 


\section{INTRODUCCIÓN}

Uno de los objetivos primordiales de un restaurante es buscar la constante mejoría de los platos ofrecidos y, obviamente, obtener mejores utilidades para el negocio. Es por esto que la Ingeniería del Menú o Menu Engineering brinda herramientas para analizar los platos que se ofrecen en la carta del restaurante y determinar si es conveniente mantenerlos o si ya esa hora de hacer un cambio.

Lo que se logra con la Ingeniería del Menú es evaluar los precios, el diseño y el contenido de la carta del restaurante y proponer las mejoras correspondientes. Para ello, clasifica los platos y bebidas para determinar cuáles de ellos son los más rentables y populares. Para aquellos platos menos populares tendremos que evaluar si corresponde mejorarlos o si es conveniente eliminarlos.

La Ingeniería del Menú nos permite optimizar la oferta gastronómica en términos de preferencias de los clientes, su evolución y las nuevas demandas.
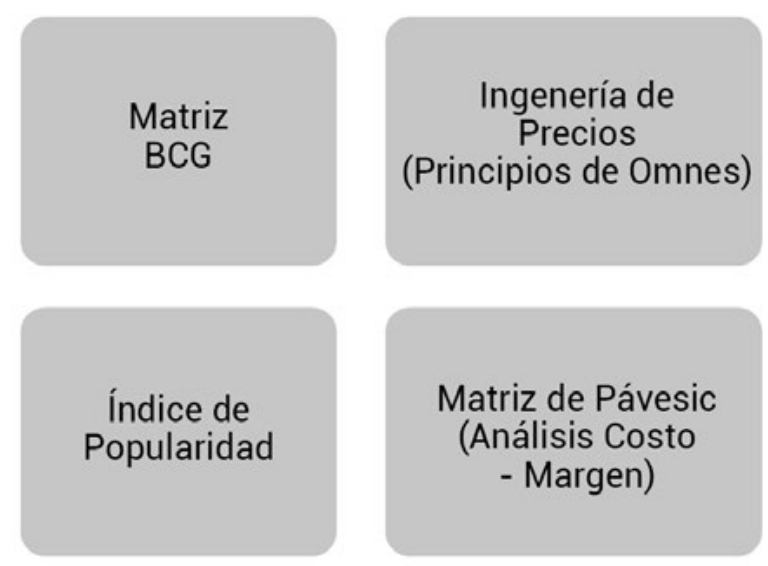

Generalmente se ha difundido mucho el uso solamente de la Matriz de Boston Consulting Group (BCG) para hablar de "Ingeniería del Menú" pero veremos que dicha herramienta es solo una de varias que a su vez se complementan.

Las otras técnicas que forman parte de la ingeniería del menú (Ingeniería de Precios, Matriz de Pávesic, Matriz de Miller, entre otras) aportan conceptos que no entrega por si sola la Matriz BCG (Basnueva et al., 2015). De ahí la necesidad de tener una visión más amplia para lograr tomar mejores decisiones en cuanto a la permanencia o no de los platos y bebidas de la carta. El objetivo final es tener mejores herramientas al momento de decidir la distribución, selección, permanencia y eliminación de los platos de la carta.

Por lo tanto, cuando hablamos de "ingeniería del menú" utilizaremos las siguientes herramientas (Figura 1):
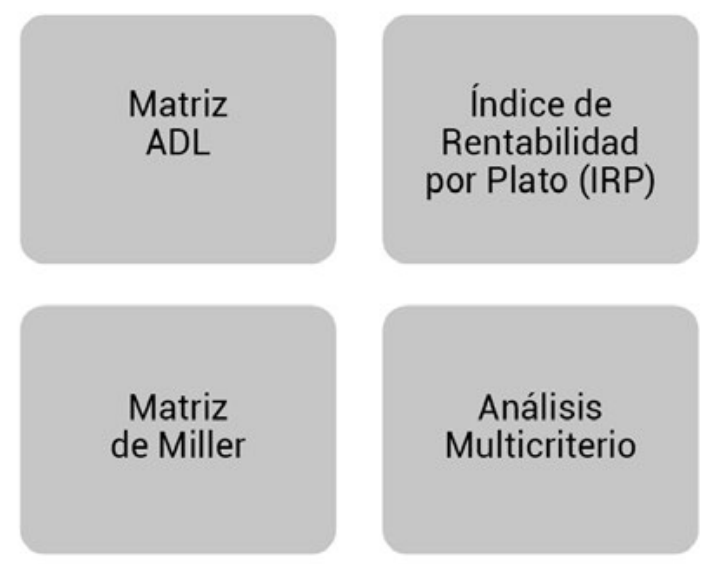

Figura 1. Herramientas que conforman la Ingeniería del menú 
Es importante señalar que todos los resultados obtenidos son propios del restaurante y no pueden ser comparados con la competencia puesto que cada negocio tiene su propia realidad.

Se recomienda aplicar estas herramientas en forma mensual e ir comparando su evolución por categoría o familia de platos y productos ofrecidos.

El Ciclo de vida del producto y la Matriz BCG

El ciclo de vida del producto muestra la evolución de un plato o producto a través de distintas etapas (tiempo) y según las ventas que realizó (cantidad vendida).

Las etapas en las cuales cada plato o producto puede pasar son: introducción, crecimiento, madurez y declinación.

Estas etapas se pueden asimilar con la clasificación que entrega la Matriz BCG. Por lo tanto, uniendo ambos conceptos nos queda lo siguiente (Figura 2).

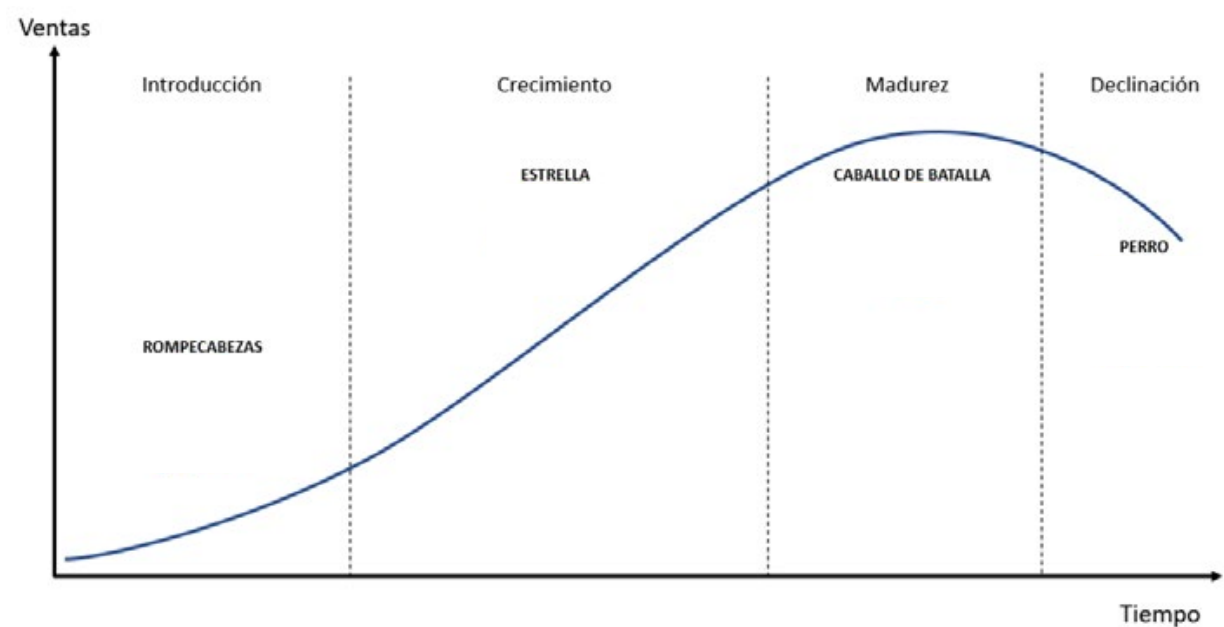

Figura 2. Paralelo entre el ciclo de vida del producto y la matriz BCG

La etapa de introducción es el lanzamiento del producto o incorporación en la carta. Las ventas son bajas en esta etapa y se invierte en publicidad y promociones. Para la Matriz BCG sería un producto impopular ("rompecabezas"). Existe mucha incertidumbre sobre la aceptación del plato o producto por parte de los comensales. El objetivo será que este plato pase a la etapa siguiente de crecimiento, es decir que se transforme en un plato estrella.

En el crecimiento, el plato ya ha ganado una buena posición y aceptación por los clientes. Posee una alta demanda y margen. Los productos estrella cumplen la misma condición en la matriz BCG. Son platos con altas ventas y grandes márgenes de utilidad.

En la etapa de madurez el plato se sigue vendiendo, pero a un menor ritmo. Según la Matriz BCG estos platos se clasifican como popular ("caballo de batalla"). Son platos con altas ventas, pero con una rentabilidad baja.

En la última etapa del ciclo del producto está la declinación. Aquí las ventas comienzan a bajar y el plato deja de 
entregar rentabilidad. La baja en las ventas se puede deber a la pérdida de interés por parte de los clientes o a la migración del consumo a nuevos platos. En la Matriz BCG estos platos reciben la categoría de perdedor ("perro"). Se debe analizar si se deben mantener -mejorando su clasificación- o si es necesario retirarlos de la carta (Carrión, 2007).

Es importante mencionar que no todos los platos o productos deben pasar obligatoriamente por cada una de las etapas. Es decir, puede suceder que un producto esté en la etapa de "introducción" y durante el tiempo pase a "declinación" directamente puesto que no fue aceptado por los clientes y por lo tanto sus ventas no crecieron. Además, algunos platos se pueden mantener siempre en la misma etapa o retroceder a una etapa anterior. Todo este análisis es a nivel interno del restaurante.

Por lo tanto, para poder analizar ambos conceptos (el ciclo de vida del producto y la matriz BCG), se aplicará la Matriz ADL para lograr este objetivo.

\section{La Matriz ADL}

La Matriz de Arthur D. Little (ADL) fue creada a mediados de los 70 y muestra la combinación del ciclo de vida del producto con la posición competitiva de la empresa. La propuesta y reestructuración que se presenta de esta matriz es hacerlo a un nivel interno del negocio.

Se combinará el ciclo de vida del producto (expresado por su margen de contribución) con las cantidades vendidas (Figura 3).

Margen de contribución

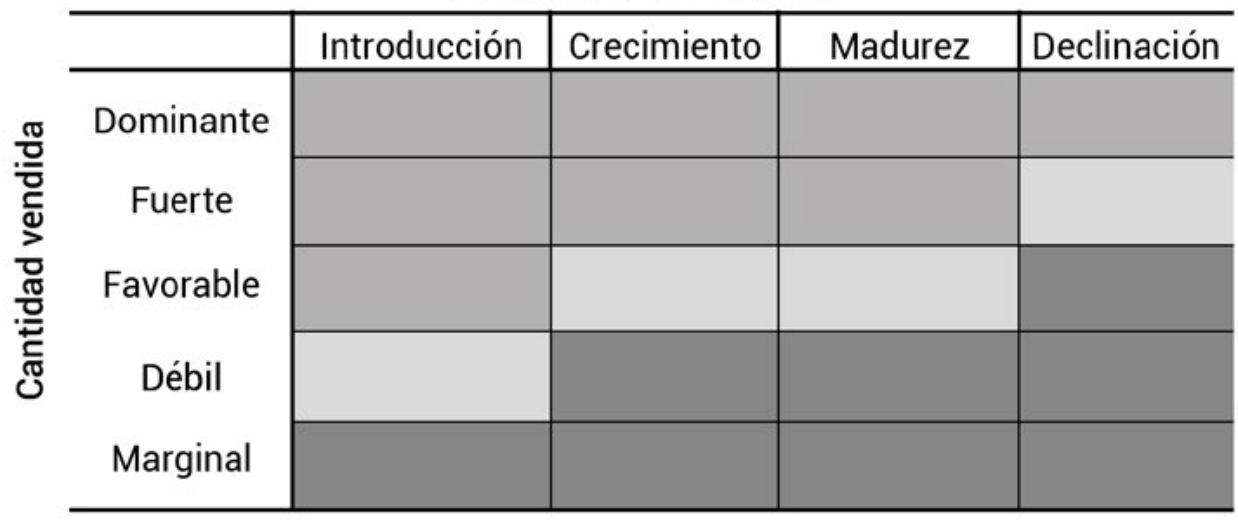

Figura 3. Matriz ADL

En el eje $X$ se representa el ciclo de vida del producto, con las etapas de: introducción, crecimiento, madurez y declinación, a través del margen de contribución.

En el eje $Y$ se representan las cantidades vendidas de los platos en cinco niveles: Dominante, fuerte, favorable, débil y marginal.

De la matriz surgirán tres zonas.

La zona verde se llama "desarrollo natural". 
Se debe mantener y potenciar la venta de los platos que se ubican en esta zona catalogados como "altos".

La zona amarilla se denomina "desarrollo selectivo". Son aquellos platos catalogados como "medios". Se debe ser selectivo en esta zona para determinar cuál de dichos platos se deben mejorar.

La última zona de color rojo se llama "abandono". Por su baja cantidad de venta y poco margen de contribución, son platos que deben ser eliminados de la carta y considerar incorporar nuevas preparaciones. Son platos que no tuvieron buena acogida por parte de los clientes o ya cumplieron su ciclo de vida y ya no son un atractivo para el público objetivo del restaurante.

\section{Metodología propuesta}

Para poder evaluar los resultados del restaurante en materia de sus preparaciones vendidas, se propone modificar el orden del ciclo de vida para poder analizar los resultados obtenidos. El orden actual que posee es: introducción, crecimiento, madurez y declinación. Este cambio es para tener un orden descendente. Por lo tanto, queda en el siguiente orden: crecimiento, introducción, madurez y declinación (Figura 4).

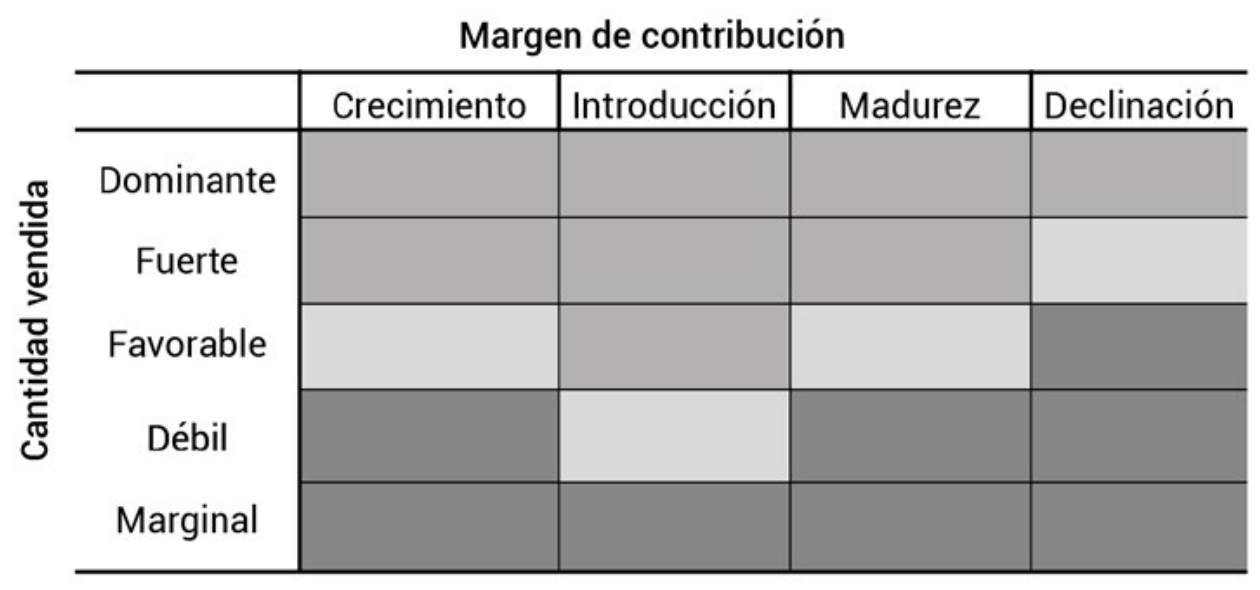

Figura 4. Matriz ADL modificada.

De esta manera se hará más fácil el análisis del ciclo de vida del producto y de la matriz ADL para poder expresarlo en una gráfica cartesiana.

\section{Aplicación de la nueva Matriz ADL}

Para poder entender mejor la aplicación de esta propuesta, veremos un ejemplo ficticio con la aplicación de esta nueva Matriz ADL.

Un restaurante cuenta con la siguiente información de sus platos de entrada (Tabla 1): 
Tabla 1. Datos de los platos de entrada

\begin{tabular}{lcccc}
\hline Entradas & $\begin{array}{c}\text { Unidades } \\
\text { vendidas }\end{array}$ & $\begin{array}{c}\text { Valor } \\
\text { venta } \\
\text { (um) }\end{array}$ & $\begin{array}{c}\text { Costo } \\
\text { unitario } \\
\text { (um) }\end{array}$ & Margen \\
\hline Plato 01 & 550 & 40 & 13 & 27 \\
Plato 02 & 280 & 45 & 14 & 31 \\
Plato 03 & 120 & 38 & 27 & 11 \\
Plato 04 & 390 & 42 & 14 & 28 \\
Plato 05 & 180 & 37 & 11 & 26 \\
Plato 06 & 530 & 25 & 8 & 17 \\
Plato 07 & 470 & 29 & 10 & 19 \\
Plato 08 & 140 & 35 & 12 & 23 \\
Plato 09 & 460 & 20 & 5 & 15 \\
Plato 10 & 110 & 30 & 13 & 17 \\
Plato 11 & 330 & 27 & 8 & 19 \\
Plato 12 & 250 & 39 & 13 & 26 \\
Plato 13 & 290 & 23 & 6 & 17 \\
Plato 14 & 90 & 36 & 15 & 21 \\
\hline
\end{tabular}

Empezaremos por definir los límites de las cantidades vendidas. Para ello, tomaremos la cantidad más alta, le restaremos la más baja y se dividirá en cinco (por tener cinco niveles).

Para ello, se utiliza la siguiente fórmula (Arthur D. Little, 1974):

\section{$\frac{\text { Cantidad más alta - Cantidad más baja }}{5}$}

El resultado es el siguiente:

$\frac{550-90}{5}=92$

Por lo tanto, la distancia y distribución de los cinco niveles de las cantidades vendidas queda de la siguiente manera (Tabla 2):

Tabla 2. Distancia y distribución de los 5 niveles de cantidades vendidas

\begin{tabular}{lcc}
\hline \multicolumn{1}{c}{ Nivel } & Desde & Hasta \\
\hline Dominante & 458 & 550 \\
Fuerte & 366 & 458 \\
Favorable & 274 & 366 \\
Débil & 182 & 274 \\
Marginal & 90 & 182 \\
\hline
\end{tabular}


Ahora definiremos los límites del ciclo de vida a través del margen de contribución de cada plato. Se toma el margen más alto, se le resta el margen más bajo y se divide en cuatro (por tener cuatro etapas del ciclo de vida).

Para ello, se utiliza la siguiente fórmula (Arthur D. Little, 1974):

Margen más alto - Margen más bajo
El resultado es el siguiente:

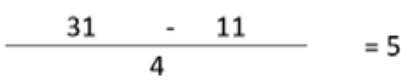

Por lo tanto, la distancia y distribución de los cuatro niveles del ciclo de vida (expresado a través del margen de contribución) queda de la siguiente manera (Tabla 3):

Tabla 3. Distancia y distribución de los 4 niveles de margen de contribución

\begin{tabular}{lcc}
\hline \multicolumn{1}{c}{ Nivel } & Desde & Hasta \\
\hline Crecimiento & 26 & 31 \\
Introducción & 21 & 26 \\
Madurez & 16 & 21 \\
Declinación & 11 & 16 \\
\hline
\end{tabular}

Los resultados obtenidos se pueden expresar en la siguiente gráfica cartesiana de la Matriz ADL modificada (Figura 5):

Matriz ADL

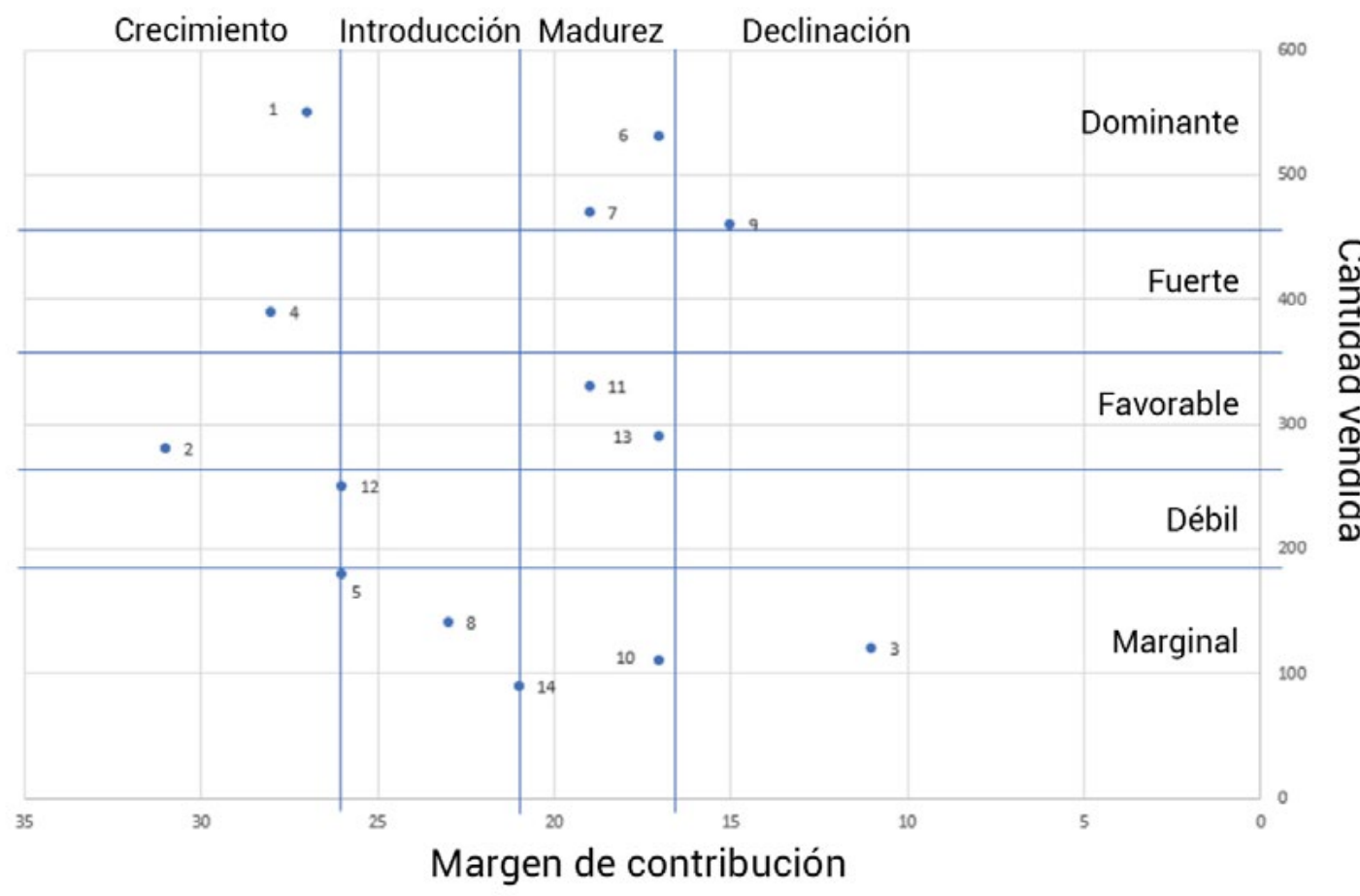

Figura 5. Gráfica cartesiana de la Matriz ADL modificada con resultados obtenidos. 
El resumen de los resultados se expresa en la Figura 6:

Margen de contribución

\begin{tabular}{|c|c|c|c|c|}
\hline & Crecimiento & Introducción & Madurez & Declinación \\
\hline Dominante & Plato 1 & & Plato 6 y 7 & Plato 9 \\
\hline Fuerte & Plato 4 & & & \\
\hline Favorable & Plato 2 & & Plato 11 y 13 & \\
\hline Débil & & Plato 12 & & \\
\hline Marginal & & Plato 5 y 8 & Plato 10 y 14 & Plato 3 \\
\hline
\end{tabular}

Figura 6. Resumen de los resultados obtenidos

\section{RESULTADOS}

Los platos que debemos mantener y potenciar serían los números: 1, 4, 6, 7 y 9 .

Analizando en forma detallada estos platos, vemos que el plato 1 es su plato "estrella". Es el mejor posicionado de todos. En segundo lugar, le sigue el plato 4 que está en la misma etapa de crecimiento, pero su cantidad vendida es "fuerte". Potenciando y promocionando más este plato 4 podrá llegar a ser "dominante".

Los platos 6 y 7 poseen una dominante cantidad de ventas, pero ya están en un nivel de madurez, es decir, son platos "populares". Son platos que la mayoría consume, pero no deja un alto margen de utilidad.

El plato 9 también posee una dominante cantidad de ventas, pero está iniciando su etapa de declinación. Se sugiere mejorar su margen (rentabilidad) para que pueda moverse a otro nivel. Se debe analizar esta situación puesto que, como se mencionó, tiene un gran potencial de ventas, pero muy baja rentabilidad.

Los platos "medios" (zona amarilla) son platos de selección, es decir, son aquellos que debemos decidir en forma selectiva su permanencia. En este ejemplo serían los platos: 2, 11, 12 y 13 .

El plato que se debería mejorar es el número 2. Se encuentra en la etapa de crecimiento y podría mejorar sus ventas realizando promociones y sugerencias por parte de los mozos. Con el plato 12 sucede algo similar y debemos analizar su permanencia con las promociones que se puedan aplicar.

Por su parte, los platos 11 y 13 son platos "populares" que están en un nivel favorable de ventas, pero si no se promocionan, se transformarán rápidamente en platos "perdedores" (declinación).

Los platos cuya eliminación debemos plantearnos serían los que se ubican en la zona roja. Estos platos son: 3, 5, 8, 10 y 14.

De estos platos, los que todavía se podrían mejorar son los números 5 y 8 . Por los resultados obtenidos, son platos que se han incorporado recientemente. Están en 
una etapa de introducción y necesitan ser más conocidos por los clientes. Esto se puede lograr obsequiando degustaciones a los comensales. Por otra parte, si son platos que estaban en la carta hace ya un tiempo, su resultado significa que por la baja cantidad vendida (marginal) son platos que no tuvieron una buena aceptación por parte de los clientes. Se debe analizar su permanencia y se deben aplicar nuevas medidas desde promociones, cambiar el nombre, incorporar o cambiar algunos ingredientes para hacerlos más atractivos.

Los platos 10 y 14 son platos que tarde o temprano se transformarán en perdedores (declinación). Sus ventas son muy bajas y son candidatos para eliminarse de la carta.

Por último, el plato 3 es el peor evaluado de toda la categoría de platos de entrada. Sus ventas son marginales y está en la etapa de declinación. Este plato debe ser eliminado de la carta.

\section{CONCLUSIONES}

La Ingeniería del Menú nos proporciona herramientas indispensables para analizar y tomar decisiones sobre la carta del restaurante. Cada una de estas herramientas permite hacer un análisis sobre dos frentes: La Matriz BCG analiza popularidad y rentabilidad; La Matriz Pávesic analiza costos y rentabilidad; La Matriz Miller analiza costos y cantidad vendida; el análisis Multicriterio es una combinación de estas tres herramientas. Por otro lado, los principios de Omnes analizan los precios de la carta (ingeniería de precios). Cada una de estas herramientas de análisis aporta algo diferente, un análisis por pares y aunque en algunos casos se repiten las variables (como el caso de la rentabilidad y el costo) son combinaciones separadas, distintos pares que brindan resultados diferentes.

La propuesta de incorporar la Matriz de Arthur D. Little (ADL), nos ayuda a observar otro par de conceptos: Las cantidades vendidas y el ciclo de vida de los productos expresado a través del margen de contribución. Si bien la Matriz ADL es una herramienta usualmente aplicada para analizar el entorno, es decir lo que sucede fuera de la empresa, se propone introducirla como herramienta de análisis interno del restaurante para evaluar su propia carta. Para ello se ha planteado modificar el orden de ciclo de vida para poder hacer el análisis en forma descendente.

La Matriz original plantea como ciclo: introducción, crecimiento, madurez y declinación. La reestructuración planteada es que el ciclo sea el siguiente: crecimiento, introducción, madurez y declinación. El hacerlo de forma descendente permite poder analizarlo en un diagrama cartesiano de ejes $X$ e $Y$, ya que visualmente sus variables se pueden interpretar con mayor facilidad. De esta manera, al adaptar la Matriz ADL a nivel interno se puede analizar la competencia entre platos de la carta del mismo restaurante y esto permite saber qué platos deben mantenerse o eliminarse de la carta en función de su demanda.

En el Programa en Excel "Ingeniería del Menú" se propuso introducir en el Análisis Multicriterio otras herramientas: el índice de popularidad e índice de rentabilidad por plato. Esta Matriz ADL reestructurada, que por sí sola es una nueva y útil herramienta de análisis, podría también formar parte del Análisis Multicriterio para una evaluación aún más completa de la carta. 


\section{REFERENCIAS BIBLIOGRÁFICAS}

Basnueva, T., Romani, B. y Espinoza, J. (2015). El menú de un restaurante. Su evaluación e incidencia en la satisfacción del cliente externo. Recuperado a partir de http://ecociencia.ecotec.edu.ec/articulo?i$\mathrm{da}=59$.

Carrión, J. (2007). Estrategia de la visión a la acción. Madrid. Segunda edición. Alfaomega Grupo editor.

Diccionario Empresarial. Matriz ADL. A partir de http://diccionarioempresarial. wolterskluwer.es/Content/Documento. aspx? params $=\mathrm{H} 4$ sIAAAAAAAEAMtMSbF $1 \mathrm{i} T$ AAASMTwMDtbLUouLMDxblwMDSONDQ3 OQQGZapUtckhIQaptWmJOcSoAkngJ6TUA $A A A=W K E$.

García, M. y Navarro, M. (2018). Inteligencia competitiva y dirección de empresas. Valencia. Primera edición. Editorial Tirant Lo Blanch.

López, M. D. (2018) Análisis de la cartera de negocios. Universidad de Alicante. Recuperado a partir de https://rua.ua.es/ dspace/bitstream/10045/23675/6/ TEMA\%205.pdf.

Molina, A. (2016). Análisis de empresas con hoja de cálculo: un caso práctico. Trabajo Fin de Grado para la obtención del título de Graduada en Administración y Dirección de Empresas (Opción Bilingüe). Recuperado a partir de https://pdfs.semanticscholar.org/ eb16/7bfa6e5c5dfe9cc43e7852853e71dcf 8d8c3.pdf. 